\title{
Binding energy of excitons in parabolic quantum wells in uniform electric and magnetic fields
}

\author{
A. Taqi, J. Diouri \\ Faculté des sciences, Département de Physique, Université Abdelmalek Essaadi, \\ BP 2121, Tétouan, Morocco \\ E-mail:abtaqi@yahoo.fr
}

\begin{abstract}
Parameterized equations have been derived using variational calculation for energy levels of Wannier excitons in 2D-parabolic quantum wells in uniform electric and magnetic fields. The formulation has been performed in the framework of the effective mass approximation and two-band model. Illustrations have been given for GaAlAsGaAs-GaAlAs and CdMnTe-CdTe-CdMnTe parabolic quantum wells. The comparison with existing data displays good agreement.
\end{abstract}

Keywords: parabolic quantum well, exciton, binding energy.

Manuscript received 27.10.11; revised manuscript received 16.12.11; accepted for publication 26.01.12; published online 29.02.12.

\section{Introduction}

Parabolic and semi-parabolic quantum well (PQW, SPQW) structures have attracted considerable interest since the end of 1980s. They were grown and investigated experimentally [1]. In comparison with rectangular quantum well (RQW), parabolic potential results in a stronger localization and equidistant energy separation of eigenvalues, as well as a rich optical spectra making $1 \mathrm{~s}, 2 \mathrm{~s}, 3 \mathrm{~s}$ exciton states and interband transitions up to $n=5$ are observable [2]. In this paper, we present a general and direct formulation of the problem of the exciton in parabolic quantum wells in uniform electric and magnetic fields, which removes tedious intermediate calculation and makes the solution of the exciton problem faster and more accessible. For the complete theory, exact solution of this problem is rather complicated because of the large number of involved effects such as valence band mixing, coupling between excitons belonging to different subbands, in addition to the anisotropy of the bands and the carrier effective mass mismatch and the dielectric mismatch. The relative importance of these effects obviously depends on the nature of the band structure of bulk materials forming the heterostructure. In the recent decade, part of this work was done on these questions, and accurate theories, mainly for RQWs, are now well established [3,4]. However, determination of exciton properties for a parabolic $2 \mathrm{D}$-confining potential can be obtained in a relatively easy way when neglecting subband and band mixing corrections. The appropriate method in this case is the commonly used variational method in 2-band model. In the work hereby, we establish, in the framework of this method, a set of advanced analytical equations suitable to calculate the energy levels of the exciton in a given 2D-confining parabolic potential. We have taken as a starting point the formalism developed by Harrison et al. [5] for the specific case of CdMnTe-CdTe-CdMnTe rectangular quantum wells, into which we have added substantial improvements. The equations were judiciously arranged and simplified as much as possible, thus reducing the stage of numerical calculation and facilitating generalization. One of the advantages of the method is that all the useful entities are expressed in terms of some elementary integrals that involve only the solution of the one-particle problem. Success of the model is illustrated by comparing our results with the available data in the cases of CdMnTe-CdTe-CdMnTe and GaAlAs-GaAsGaAlAs PQWs for the ground ( $X_{1 s}$ ) exciton states [6].

\section{The model}

Consider a heterostructure consisting of a single quantum well $(\mathrm{QW})$ of type $\mathrm{I}$ in the $z$-direction. The confining potential is of a parabolic shape, say $V_{e}\left(z_{e}\right)$ for electrons and $V_{h}\left(z_{h}\right)$ for holes. 
Uniform electric $\vec{F}$ and magnetic $\vec{B}$ fields are considered to be parallel to the $z$-direction. Within the framework of the effective mass approximation, the Hamiltonian of an electron-hole system under the magnetic and electric fields, for the ground state $X_{1 \mathrm{~s}}$, is as follows:

$$
H=H_{e}+H_{h}+H_{e x}
$$

with

$$
\begin{aligned}
& H_{e}=-\frac{\hbar^{2}}{2 m_{e}} \frac{\partial^{2}}{\partial z_{e}^{2}}+V_{e}\left(z_{e}\right)+e F z_{e}, \\
& H_{h}=-\frac{\hbar^{2}}{2 m_{h}} \frac{\partial^{2}}{\partial z_{h}^{2}}+V_{h}\left(z_{h}\right)-e F z_{h}, \\
& H_{e x}(\rho, r)=-\frac{\hbar^{2}}{2 \mu}\left(\frac{1}{\rho} \frac{\partial}{\partial \rho}+\frac{\partial^{2}}{\partial \rho^{2}}\right)+\frac{e^{2}}{8 \mu} B^{2} \rho^{2}-\frac{e^{2}}{\varepsilon r},
\end{aligned}
$$

where $\rho=\left|\vec{\rho}_{e}-\vec{\rho}_{h}\right|$ is the relative distance between electron and hole in the $x-y$ plane, $\varepsilon$ represents the dielectric constant, $\quad r=\sqrt{\rho^{2}+\left(z_{e}-z_{h}\right)^{2}}, \quad H_{e}\left(H_{h}\right)$ describes the motion of the electron (hole) in a constant electric field $F$ along $z$-axis with the effective mass $m_{e}\left(m_{h}\right)$, and $H_{e x}$ describes the in-plane relative motion with the reduced mass $\mu$.

Exciton eigenenergies $E$ and wavefunctions $\psi$ are determined by minimizing the expected value $H$ with respect to a suitable variational parameter:

$$
E=\min \langle\psi|H| \psi\rangle /\langle\psi \mid \psi\rangle \text {. }
$$

The trial wavefunction $\psi$ is chosen as $\psi=\psi_{e} \psi_{h} \phi$,

where $\phi$ is the coupling factor that will be chosen to conveniently describe the exciton state. Thus, we take

$$
\phi_{1 s}=\exp \left(-\frac{\sqrt{\rho^{2}+a^{2}}}{\lambda}\right)
$$

where $\lambda$ is the variational parameter and $a=\left|z_{e}-z_{h}\right|$.

The first Landau levels are given by $E_{0}=\gamma R$,

where the dimensionless parameter $\gamma=\hbar \omega_{c} / 2 R$ and $\omega_{c}=\frac{e B}{\mu}$ is the cyclotron frequency, the scaling quantity $R$ is the effective Rydberg defined by $R=\frac{\hbar^{2}}{2 \mu\left(a^{*}\right)^{2}}$ and $a^{*}=\frac{\varepsilon \hbar^{2}}{e^{2} \mu}$ is the Bohr effective radius.

Then, for non-vanishing magnetic field, the binding energy $E_{B}$ is given by
$E_{B}=E_{e}+E_{h}+E_{0}-E$,

where $E_{e}$ and $E_{h}$ are solutions of the one-particle Hamiltonian in a constant electric field $H_{i} \psi_{i}=E_{i} \psi_{i}$.

The expression (5) is now to be evaluated in order to derive $E(\lambda)$ and then $E_{B}(\lambda)$. Substituting (6) and (7) into (5) and performing integration, the parameterized binding energy reads as

$$
\begin{aligned}
& E_{B}(\lambda)=R\left(\frac{a^{*}}{\lambda}\right)^{2}\left[-1+\frac{4 \lambda}{\lambda I_{0}+2 I_{1}}\left((1-\sigma) L+\frac{\lambda}{a^{*}} I_{0}\right)\right]+\gamma \mathrm{R}- \\
& -R\left(\frac{\lambda}{a^{*}}\right)^{2} \frac{1}{4} \gamma^{2}\left[\frac{3}{2}+\frac{2 I_{2}}{\lambda^{2} I_{0}+2 \lambda I_{1}}\right],
\end{aligned}
$$

where the entities $I_{n}(\lambda)$ and $L$ are given in appendix (equations (A2), (A10)), and $\sigma$ is the reduced mass ratio given by $\sigma=\frac{\mu}{\mu_{z}}$ with $\frac{1}{\mu_{z}}=\frac{1}{m_{e}}+\frac{1}{m_{h}}$.

\section{Results and discussions}

In this work, we apply the model developed in the section 2 to calculate binding energy of excitons in a single infinitely deep wide parabolic well (WPW). Curiously, this model seems to be much more adapted to PQWs making this solution quasi-analytical. The studied structure consists in a type I heterostructure with parabolic well for electrons and holes, described by $V_{i}\left(z_{i}\right)=\frac{\alpha_{i}^{2}}{2 m_{i}} z_{i}^{2}$, where $\alpha_{i}=\frac{2}{L_{w}} \sqrt{2 m_{i} U_{i}}, U_{i}$ being the discontinuity of the band edge between the centre of the well and its outermost layers. Furthermore, it is well known that when the dimensionless quantity $\Omega_{i}=\frac{\alpha_{i} L_{w}^{2}}{4}$ is larger than approximately 3 , the difference between the ground state energies of the finite and the infinite quantum wells is negligible [8]. Then, the ground state solutions of one particle problem for the parabolic well in the absence of electric field are those of the harmonic oscillator, namely: $\quad \psi_{i}\left(z_{i}\right) \propto \exp \left(-\frac{1}{2} \alpha_{i} z_{i}^{2}\right) \quad$ with $E_{i}=\frac{\alpha_{i}}{2 m_{i}}$. In addition, for current values of structure parameters, the function $p(a)$ reduces, in this particular case, to the following simplified form:

$$
p(a)=2 \sqrt{\frac{\pi}{\alpha_{e}+\alpha_{h}}} \exp \left(-\alpha a^{2}\right), \alpha=\frac{\alpha_{e} \alpha_{h}}{\alpha_{e}+\alpha_{h}},
$$

which gives for $I_{n}$ integrals the iterative relation:

$$
I_{n+2}=\frac{n+1}{2 \alpha} I_{n}-\frac{1}{\alpha \lambda} I_{n+1}
$$




\subsection{In the absence of magnetic and electric fields}

To test the validity of equation (10), we have applied our model first in the absence of magnetic and electric fields, to calculate the binding energy of $\mathrm{HX}$ excitons for $\mathrm{Ga}_{0.7} \mathrm{Al}_{0.3} \mathrm{As}-\mathrm{GaAs}-\mathrm{Ga}_{0.7} \mathrm{Al}_{0.3} \mathrm{As}$ and $\mathrm{Cd}_{0.22} \mathrm{Mn}_{0.78} \mathrm{Te}-$ $\mathrm{CdTe}-\mathrm{Cd}_{0.22} \mathrm{Mn}_{0.78} \mathrm{Te}$. By way of comparison, we have referred to the article of Rusin et al. [6] and used the same parameters therein. In this work, the authors have used the so-called "effective variational method", in which the form of the trial functions is subject to variation. The results obtained using this method are very close to ours, as it is shown in Fig. 1, for the whole range of $L_{w}$ values and for both structures. However, energy values calculated using our method are found to be slightly higher and more close to the experimental values that are reported in the same article.

\subsection{Magnetic field effect}

In Fig. 2 we show, for the intensity of electric field $F=0$, the variation of the exciton binding energy $E_{B}$ in a $\mathrm{Ga}_{0.7} \mathrm{Al}_{0.3} \mathrm{As}-\mathrm{GaAs}-\mathrm{Ga}_{0.7} \mathrm{Al}_{0.3} \mathrm{As}$ PQW for $1 \mathrm{~s}$ states of heavy-hole excitons as a function of well width $L_{W}$ for $B=0, B=5 \mathrm{~T}$ and $B=15 \mathrm{~T}$. It is obvious from the figure that, for a given magnetic field, the exciton binding energy decreases as $L_{W}$ increases. It takes place because with increasing $L_{W}$ the probability of finding the electron and hole in the same plane decreases. Increasing the magnetic field enhances the confinement of particles in the $x-y$ plane. Therefore, the exciton binding energy increases with magnetic field. Similar behavior was reported by E. Kasapoglu et al. [8] for rectangular quantum wells.

\subsection{Electric field effect}

As an original application of the model, we have examined the case of a $z$-applied electric field in PQW. Solution of the problem is immediate and needs no further calculation. Indeed, computation is performed by using the same numerical program where the parameters were modified in the suitable way. In fact, it is easy to demonstrate that the additional term, in the Hamiltonian, arising from the applied electric field $F, \pm e F z_{e, h}$, leads to the following expression for $p(a)$ :

$$
p_{F}(a)=\frac{1}{2}[p(a+\delta)+p(a-\delta)]
$$

where $\delta=\frac{e F L_{w}^{2}}{8}\left(\frac{1}{U_{e}}+\frac{1}{U_{h}}\right)$ and $p(a)$ given by Eq.

Hence, this solution consists of a simple translation of the parameter $a$ by amount $\pm \delta$. In Fig. 3, for vanishing magnetic field, ground state binding energies of excitons in the presence of an electric field are plotted as a function of the well width for the same structures studied in the previous section for $F=20,100$ and $200 \mathrm{kV} / \mathrm{cm}$. In this figure, one can observe that the effect of electric field is significant for all the well width values. This effect results in decreasing energy with a more important rate than that observed for $E_{B}\left(L_{w}\right)$. That indicates the degree of competition between the electric field effect and the confinement. For narrow wells the curvature of $E_{B}^{F}\left(L_{w}\right)$ is of parabolic shape, revealing a quadratic Stark shift. Similar trends have been observed for excitons in parabolic quantum dots [10]. One of the differences between these two systems is that in PQDs the slope of decreasing of $E_{B}^{F}\left(L_{w}\right)$ for narrow size is much larger than in PQWs even for weak field intensities. Furthermore, in the case of PQWs, calculation shows a priori that, for sufficiently large electric field intensities $(F>200 \mathrm{kV} / \mathrm{cm})$, the energy becomes very sensitive to the electric field effect even for narrow wells. It decreases abruptly when well width increases.

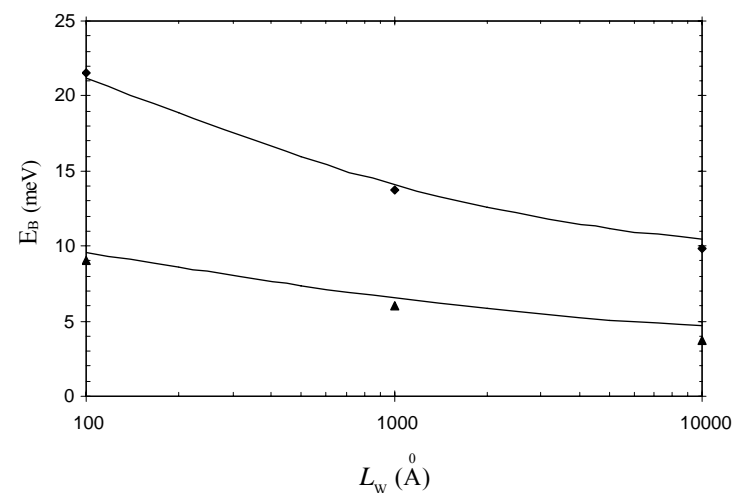

Fig. 1. Variations of the binding energy of $1 \mathrm{~s}$ heavy hole exciton in $\mathrm{GaAs}-\mathrm{Ga}_{0.7} \mathrm{Al}_{0.3} \mathrm{As}-\mathrm{GaAs}$ (down) and $\mathrm{CdTe}-$ $\mathrm{Cd}_{0.22} \mathrm{Mn}_{0.78} \mathrm{Te}$ (up) parabolic quantum wells on the well width in the absence of magnetic and electric fields. Triangles and squares refer to the results obtained by Rusin T.M. [6] using the same parameters.

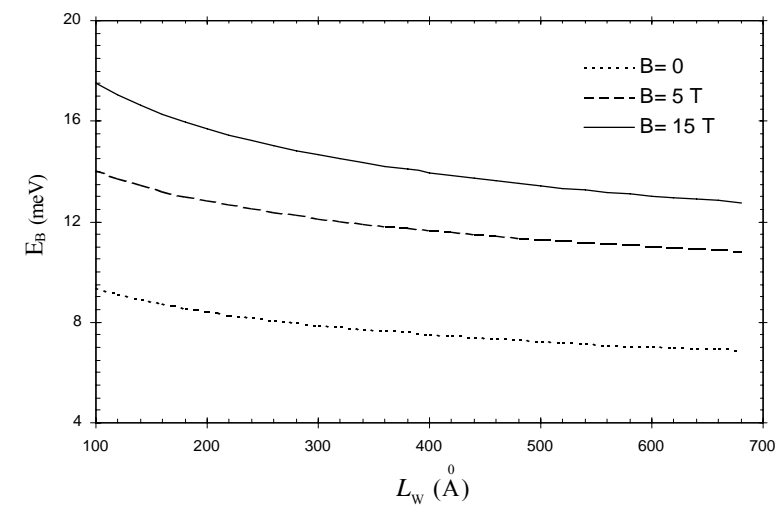

Fig. 2. Binding energy, as a function of the well width, of 1s heavy hole exciton in GaAs- $\mathrm{Ga}_{0.7} \mathrm{Al}_{0.3} \mathrm{As}-\mathrm{GaAs}$ parabolic quantum well. The calculation is done in the absence of electric field for three values of the magnetic field: $B=0$, $B=5 \mathrm{~T}$ and $15 \mathrm{~T}$, respectively, from bottom to top. 


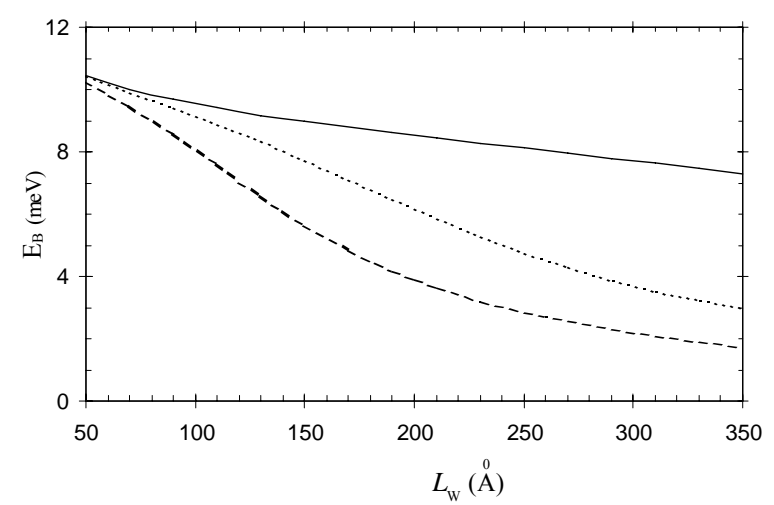

Fig. 3. Dependence of the binding energy of $1 \mathrm{~s}$ exciton in the same structures as in Fig. 1 on the well width in the absence of magnetic field for three values of the electric field: $F=20 \mathrm{kV} / \mathrm{cm}$ (solid line), $100 \mathrm{kV} / \mathrm{cm}$ (dotted line) and $200 \mathrm{kV} / \mathrm{cm}$ (dashed line).

\subsection{Electric and magnetic field effects}

Fig. 4 illustrates the dependence of the binding energy of the exciton as a function of well width in the presence of electric and magnetic fields. In general, the binding energy decreases with increasing the well width. For well widths ranging from 100 up to $200 \AA$, the effects observed are those of the magnetic field and confinement, the electric field has no influence. The magnetic field does not change behavior of the curve of $E_{B}$, but increases the energy values. For the well widths $\left(L_{w}>200 \AA\right)$, the application of an electric field changes the rate of decrease of $E_{B}$. This rate is almost constant for the well widths between 200 and $400 \AA$ and increases significantly with the magnetic field for the well widths $\left(L_{w}>400 \AA\right)$. We interpret this feature as a result of competition between the effect of electric field and the effect of confinement for $200 \AA<L_{w}<400 \AA$, and as a result of competition between the effect of electric field and magnetic field effect for $L_{w}>400 \AA$.

\section{Conclusion}

In conclusion, using a variational calculation in 2-band approximation, we have established a parameterized equation to be used for calculating exciton properties in 2D-parabolic quantum wells. First, we have tested the validity of the equation by comparing our results with calculations available in the literature for GaAs-GaAlAs and $\mathrm{CdTe}-\mathrm{CdMnTe}$ parabolic quantum wells. Starting with the success of this comparison, we have applied the model to calculate the effect of constant magnetic and electric fields parallel to the growth direction on the binding energy GaAs-GaAlAs parabolic quantum wells. And finally, let's note that the perspectives of implementing the model developed in this paper remain widely open. Owing to its simplicity, it can be extended

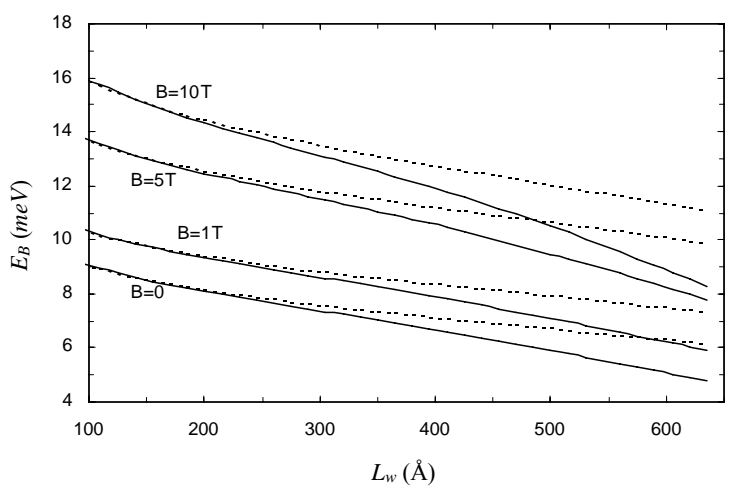

Fig. 4. Variation of the binding energy of excitons $\mathrm{HX}(1 \mathrm{~s})$ in a PQW GaAs- $\mathrm{Ga}_{0.6} \mathrm{Al}_{0.4} \mathrm{As}$ as a function of the well width for different values of the magnetic field and two electric field values $F=10 \mathrm{kV} / \mathrm{cm}$ (dashed curves) and $F=20 \mathrm{kV} / \mathrm{cm}$ (solid curves).

without difficulty to many types of applications such as exploring new potential profiles of the quantum wells, investigating higher excited states of excitons and calculating transitions energies and oscillator strength for these states, examining electric and magnetic field effects, etc.

\section{Appendix}

I) The entities $I_{n}(\lambda)$ involved in equation (10) are defined as follows [10]:

$I_{n}(\lambda)=\iint_{-\infty}^{+\infty} \psi_{e}^{2}\left(z_{e}\right) \psi_{h}^{2}\left(z_{h}\right)\left|z_{e}-z_{h}\right|^{n} e^{-\frac{2||_{e}-z_{h} \mid}{\lambda}} d z_{e} d z_{h}$

and may be written as

$I_{n}(\lambda)=\int_{0}^{\infty} p(a) e^{-\frac{2}{\lambda} a} a^{n} d a$,

where $p(a)$ represents the uncorrelated probability of finding electron and hole separated by a distance $a[5]$,

$p(a)=\int_{-\infty}^{+\infty}\left[\psi_{e}^{2}(z+a) \psi_{h}^{2}(z)+\psi_{h}^{2}(z+a) \psi_{e}^{2}(z)\right] d z$.

$I_{n}(\lambda)$ integrals satisfy two interesting iterative relations which may be obtained as follows. Taking derivative of (A1) with respect to $\lambda$, gives

$I_{n+1}=\frac{\lambda^{2}}{2} \frac{d I_{n}}{d \lambda}$

and performing integration by parts in (A2) yields

$I_{n}=n \frac{\lambda}{2} I_{n-1}+\frac{\lambda}{2} \int_{0}^{\infty} a^{n} \frac{\partial p}{\partial a} e^{-\frac{2}{\lambda} a} d a, n \geq 1$. 
II) Substituting equations (6) and (7) into (5) and performing integration, the denominator in equation (5) is given by

$\langle\psi \mid \psi\rangle=\mathfrak{I}(F)$,

where the operator $\mathfrak{I}$ is defined as:

$\mathfrak{I}(f)=\int_{0}^{\infty} p(a) f(a) d a$.

The numerator in equation (5) reduces to

$$
\begin{aligned}
& \langle\psi|H| \psi\rangle=\left(E_{e}+E_{h}\right) \mathfrak{I}(F)+\frac{h^{2}}{2 \mu_{z}} \mathfrak{I}(G)- \\
& -\frac{h^{2}}{2 \mu} \mathfrak{I}(J)-\frac{e^{2}}{\varepsilon} \mathfrak{I}(K)+\frac{e^{2}}{8 \mu} B^{2} \mathfrak{I}(P)
\end{aligned}
$$

and the entities $F, G, J, K, P$ are given by

$$
\begin{aligned}
& F(a)=2 \pi \int_{0}^{\infty} \phi^{2}(\rho, a) \rho d \rho, \\
& G(a)=2 \pi \int_{0}^{\infty}\left|\frac{\partial \phi}{\partial z_{e}}\right|^{2} \rho d \rho, \\
& P(a)=2 \pi \int_{0}^{\infty} \phi^{2}(\rho, a) \rho^{3} d \rho, \\
& J(a)=2 \pi \int_{0}^{\infty} \phi\left[\frac{1}{\rho} \frac{\partial}{\partial \rho}+\frac{\partial^{2}}{\partial \rho^{2}}\right] \phi \rho d \rho, \\
& K(a)=2 \pi \int_{0}^{\infty} \frac{1}{r} \phi^{2} \rho d \rho, \quad r=\sqrt{\rho^{2}+a^{2}} .
\end{aligned}
$$

In the same way, $L$, appearing in equation (10) is given by

$$
L=\frac{a^{2}}{\lambda^{2}} \mathfrak{J}\left(E_{i}\left(\frac{2 a}{\lambda}\right)\right), E_{i}(x)=\int_{x}^{\infty} \frac{e^{-t}}{t} d t
$$

\section{References}

1. H.M. Cheong, J.H. Burnett, W. Paul, P.F. Hopkins and A.C. Gossard // Phys. Rev. B, 49(10), p. 444 (1994).

2. F. Kyrychenko and J. Kossut // Semicond. Sci. Technol. 13, p. 1076-1079 (1998).

3. L.C. Andreani and A. Pasquarello // Phys. Rev. B, 42, p. 8928 (1990).

4. R. Winkler // Phys. Rev. B, 51, p. 14395 (1995).

5. P. Harrison, T. Piorek, W.E. Hagston and T. Stirner // Superlatt. and Microstructures, 20, p. 45 (1996).

6. T.M. Rusin // J. Phys.: Condens. Matter. 12, p. 575-587 (2000).

7. Yuen Wu-Pen // Phys. Rev. B, 48, p. 17316 (1993).

8. E. Kasapoglu, H. Sari, N. Balkan, I. Sokmen and Y. Ergun // Semicond. Sci. Technol. 15, p. 219-224 (2000)

9. S. Jaziri, R. Bernaceur // Semicond. Sci. Technol. 9, p. 1775 (1994).

10. J. Diouri, A. Taqi, A. El Haddad, M. Katih and E. Feddi // Semicond. Sci. Technol. 18, p. 377 (2003). 